\title{
ELLIPTICITY AND REGULARITY FOR PERIODIC NONLINEAR EQUATIONS
}

\author{
ROBERT A. ADAMS
}

The composition of two strongly elliptic linear operators with suitably differentiable coefficients is also strongly elliptic. This fact has been used [3, pp. 178-181] to yield a particularly simple proof of the regularity of weak solutions of periodic strongly elliptic linear equations. In this paper we prove a similar regularity theorem for periodic nonlinear equations under the assumption of strong ellipticity for the composition of the given nonlinear operator with certain linear operators.

Let $A$ be a nonlinear differential operator of order $2 m$ given by

$$
A u(x)=\sum_{|\alpha| \leqq m} D^{\alpha} a_{\alpha}(x, \xi(u)(x))
$$

where $\xi=\left(\xi_{\alpha}\right)_{|\alpha| \leq m}$ and $\xi_{\alpha}(u) \equiv D^{\alpha} u$. Here, as usual, $x=\left(x_{1}, \cdots, x_{n}\right)$; $\alpha=\left(\alpha_{1}, \cdots, \alpha_{n}\right) ;|\alpha|=\alpha_{1}+\cdots+\alpha_{n} ; D^{\alpha}=D_{1}^{\alpha_{1}} \cdots D_{n}^{\alpha_{n}}$, the $\alpha_{j}$ being nonnegative integers and $D_{j}=i^{-1} \partial / \partial x_{j}, i^{2}=-1$. Denote by $P$ the class of all functions on $R_{n}$ periodic with period unity in each variable. We assume throughout that $a_{\alpha}(\cdot, \xi) \in P$. If $Q$ denotes a cube of unit edge with edges parallel to the coordinate axes in $R_{n}$ we define the standard Dirichlet form for $A$ :

$$
a(u, v)=\sum_{|\alpha| \leqq m} \int_{Q} a_{\alpha}(x, \xi(u)(x)) \overline{D^{\alpha} v(x)} d x, \quad u, v \in P .
$$

Let $H_{m}$ denote the Hilbert space obtained by completing in the topology generated by the inner product

$$
[u, v]_{m}=\sum_{|\alpha| \leq m} \int_{Q} D^{\alpha} u(x) \overline{D^{\alpha} v(x)} d x
$$

the class $P \cap C^{\infty}\left(R_{n}\right)$ of all possibly complex-valued functions which are periodic with period unity in each coordinate and infinitely differentiable on $R_{n}$. We denote $\|u\|_{m}=(u, u)^{1 / 2}$. The dual of $H_{m}$ with respect to the $L_{2}$ inner product $[u, v]_{0}$ is denoted $H_{-m}$. Properties of these spaces are discussed in [1, pp. 165-169]. If $f \in H_{k}$, then $D^{\alpha} f \in H_{k-|\alpha|}$ for any distribution derivative $D^{\alpha}$. Given $f \in H_{k}, k \geqq-m$, $u$ is said to be a periodic weak solution of the equation $A u=f$ if $u \in H_{m}$ and for each $v \in H_{m}$

Received by the editors September 19, 1967. 


$$
a(u, v)=\langle f, v\rangle
$$

where $\langle\cdot, \cdot\rangle$ denotes the pairing between $H_{-m}$ and $H_{m}$.

The operator $A$ will be called elliptic of class $E_{m}$ if the following three conditions are satisfied:

(i) For each $r \geqq 0$ there exists a number $g(r)$ such that for all $u$, $v \in H_{m}$

$$
|a(u, v)| \leqq g\left(\|u\|_{m}\right)\|v\|_{m} .
$$

(ii) If $u_{k} \rightarrow u$ in $H_{m}$, then $a\left(u_{k}, v\right) \rightarrow a(u, v)$ for all $v$ in $H_{m}$.

(iii) There exists a constant $c>0$ such that for all $u, v \in H_{m}$

$$
|a(u, u-v)-a(v, u-v)| \geqq c\|u-v\|_{m}^{2} \text {. }
$$

For a given integer $k \geqq 0, A$ will be called elliptic of class $E_{m, k}$ if for each integer $j$ with $0 \leqq j \leqq k$ the operator $A_{j}=L^{j} A$ of order $2 m+2 j$ is elliptic of class $E_{m+j}$ where $L=1-\Delta=1+\sum_{j=1}^{n} D_{j}^{2}$. (Note that if the coefficients $a_{\alpha}$ are sufficiently differentiable then $A_{j}$ is an operator formally of type (1). If $A$ is linear with $C^{\infty}$ coefficients and is elliptic of class $E_{m}$, then it is also elliptic of class $E_{m, \infty}$.)

The existence of a periodic weak solution of $A u=f$ for $f \in H_{k}$, $k \geqq-m$ follows immediately for $A$ elliptic of class $E_{m}$ from the nonlinear extension of the Lax-Milgram theorem due to Zarantonello and Browder [2]. Specifically, (i) implies that there exists an operator $T$ mapping $H_{m}$ into itself such that $a(u, v)=[T u, v]_{m}$ for all $u, v \in H_{m}$; (ii) shows that $T$ is demicontinuous (continuous from the strong to the weak topology of $H_{m}$ ) and (iii) shows, by the above-mentioned theorem, that $T$ is one-to-one onto $H_{m}$ and has a continuous inverse. Also $|\langle f, v\rangle| \leqq\|f\|_{-m}\|v\|_{m}$ so that $\langle f, v\rangle=\left[f_{0}, v\right]_{m}$ where $f_{0} \in H_{m}$ is the projection of $f$ onto $H_{m}$. A weak solution of $A u=f$ is given by $u$ $=T^{-1} f_{0}$.

Of more interest is the following regularity

TheOREM. Let $u \in H_{m}$ be a periodic weak solution of $A u=f$ where $f \in H_{k}, k \geqq-m$. If there exists a constant $\lambda$ such that the operator $A^{\prime}=A+\lambda L^{m-1}$ is elliptic of class $E_{m, m+k}$, then $u \in H_{2 m+k}$.

Proof. It is sufficient to prove the theorem for the case $\lambda=0$ for, assuming this already done and noting that if $u$ is a periodic weak solution of $A u=f$ it is also a periodic weak solution of $A^{\prime} u=f^{\prime}$ where $f^{\prime}=f+\lambda L^{m-1} u$, we have $f^{\prime} \in H_{p}$ where $p=\min (k,-m+2)$. Since $A^{\prime}$ satisfies the condition of the theorem with $\lambda=0$ it follows that $u \in H_{2 m+p}$, whence $f^{\prime} \in H_{q}$ where $q=\min (k,-m+4)$. Hence $u \in H_{2 m+q}$. Continuing in this way we obtain $u \in H_{2 m+k}$. 
We suppose therefore that $\lambda=0$ and $A$ is elliptic of class $E_{m, m+k}$. As in the existence argument above there exist, for $0 \leqq j \leqq m+k$, demicontinuous, continuously invertible operators $T_{j}$ mapping $H_{m+j}$ oneto-one onto itself such that for all $u, v \in H_{m+j}$

$$
a_{j}(u, v)=\left[T_{j} u, v\right]_{m+j},
$$

where $a_{j}$ is the Dirichlet form of $A_{j}$. Similarly, by the Lax-Milgram theorem, there exist homeomorphisms $V_{j}$ mapping $H_{m+j}$ onto itself such that for all $u, v \in H_{m+j}$

$$
l_{j}(u, v)=\left[V_{j} u, v\right]_{m+j}
$$

where $l_{j}$ is the Dirichlet form for $L^{m+j}$. Hence, for $0 \leqq j \leqq m+k, S_{j}$ $=v_{j}^{-1} T_{j}$ is a one-to-one mapping of $H_{m+j}$ onto itself which satisfies for all $u, v \in H_{m+j}$

$$
a_{j}(u, v)=l_{j}\left(S_{j} u, v\right) .
$$

On the other hand, by integrating by parts and using periodicity, we see that for all $\phi, \psi \in P \cap C^{\infty}\left(R_{n}\right)$,

$$
a_{j}(\phi, \psi)=a_{0}\left(\phi, L^{j} \psi\right)=l_{0}\left(S_{0} \phi, L^{j} \psi\right)=l_{j}\left(S_{0} \phi, \psi\right),
$$

whence $w=S_{j} \phi-S_{0 \phi} \phi$ is a periodic weak solution of the linear equation $L^{m+j} w=0$ and as such is the null element of $H_{m+j}$. By completion $S_{j}$ is the restriction of $S_{0}$ to $H_{m+j}$ and so $S_{0}$ maps $H_{m+j}$ one-to-one onto itself.

Now since $u$ is a periodic weak solution of $A u=f, f \in H_{k}$, it follows that $v=S_{0} u$ is a periodic weak solution of the linear equation $L^{m_{v}}=f$ which by Friedrichs' theorem (periodic case) [3, pp. 178-181] belongs to $H_{2 m+k}$. Since $S_{0}$ maps $H_{2 m+k}$ one-to-one onto itself $u \in H_{2 m+k}$ as required.

\section{REFERENCES}

1. L. Bers, F. John and M. Schechter, Partial differential equations, Interscience, New York, 1964.

2. F. Browder, Remarks on nonlinear functional equations, Proc. Nat. Acad. Sci. U. S. A. 51 (1964), 985-989.

3. K. Yosida, Functional analysis, Academic Press, New York, 1965.

The University of British Columbia, Vancouver 